EPiC Series in Computing
Volume 62, 2019, Pages 124-144
Sumo User Conference 2019

\title{
From Automated to Manual - Modeling Control Transitions with SUMO
}

\author{
Leonhard Lücken ${ }^{1 *}$, Evangelos Mintsis ${ }^{2}$, Kallirroi N. Porfyri², Robert \\ Alms ${ }^{1}$, Yun-Pang Flötteröd ${ }^{1}$, Dimitris Koutras ${ }^{2}$ \\ ${ }^{1}$ German Aerospace Center (DLR), Institute of Transportation Systems, Rutherfordstraße 2, 12489 \\ Berlin, Germany \\ ${ }^{2}$ Centre for Research and Technology Hellas, Hellenic Institute of Transport, 6th Km Charilaou - \\ Thermi Rd., 57001 Thermi, Thessaloniki, Greece \\ *leonhard.luecken@dlr.de
}

\begin{abstract}
Transitions of Control (ToC) play an important role in the simulative impact assessment of automated driving because they may represent major perturbations of smooth and safe traffic operation. The drivers' efforts to take back control from the automation are accompanied by a change of driving behavior and may lead to increased error rates, altered headways, safety critical situations, and, in the case of a failing takeover, even to minimum risk maneuvers. In this work we present modeling approaches for these processes, which have been introduced into SUMO recently in the framework of the TransAID project. Further, we discuss the results of an evaluation of some hierarchical traffic management (TM) procedures devised to ameliorate related disturbances in transition areas, i.e., zones of increased probability for the automation to request a $\mathrm{ToC}$.
\end{abstract}

\section{Introduction}

The automation of the dynamic driving task is foreseen to revolutionize the existing paradigm in the road transportation sector. In the past two decades, driving automation systems of different capabilities, ranging between semi-autonomy and full autonomy, were researched and developed. Currently, many production-series vehicles are equipped with Advanced Driver Assistance Systems (ADAS), that can undertake different aspects (longitudinal, lateral or both) of the dynamic driving task (ERTRAC Working Group, 2017). Adaptive Cruise Control (ACC), Lane Keeping Assist (LKA), and Forward Collision Warning (FCW) are among the most common ADAS that market available vehicles are manned with. Recently, conditional automation (SAE Level 3) was also introduced into 
specific market ready passenger cars in the form of Traffic Jam Assist. Although conditionally automated driving systems are designed to monitor the road environment, the vehicle operator still remains the failsafe in case of automation disengagement. However, the automotive industry and selfdriving technology providers have announced that highly automated vehicles (AVs) should be expected to enter the roads on the dawn of the upcoming decade. These vehicles will explicitly operate within confined geographical areas named Operational Design Domains (ODDs). Moreover, they will be capable to control the fallback performance of the dynamic driving task if the driver is unresponsive to take-over requests (TORs) when system-initiated automation disengagements commence. Thus, vehicle automation will gradually alter road traffic composition in the next decades (PTOLEMUS Consulting Group, 2017) and significantly impact the fronts of traffic safety and efficiency.

Evidence based on field operational testing (controlled and real traffic experiments) of automated and autonomous vehicles indicated that automation disengagements can result from a multitude of reasons. System failures, human factors, complex traffic situations and improper infrastructure (e.g. ambiguous or absent lane markings) were proven as the primary causes of downward (from automated to manual driving) transitions of vehicle control (Favarò, Eurich, \& Nader, 2018).

ToCs are expected to exert a profound impact on traffic operations due to erratic vehicle behavior and potential minimum risk maneuvers (MRMs). This phenomenon will be amplified in the presence of mixed traffic (coexistence of manual and automated/autonomous vehicles on the roads) due to heterogeneous vehicle behavior that will induce complex vehicle interactions. Although future autonomous vehicles are designed to predict the intentions and imminent actions of other road users even in the absence of connectivity (Bansal, Krizhevsky, \& Ogale, 2018), developers of self-driving technology expect that the long tail of edge cases that vehicle automation will be incapable to cope with will prevent a complete release of the human driver from fallback responsibilities for a long time. Thus, the investigation of the effects of ToC/MRM on road traffic is of prominent significance.

ACC activation and deactivation was thoroughly investigated by many previous studies (Pauwelussen \& Feenstra, 2010; Pauwelussen \& Minderhoud, 2008; Viti, Hoogendoorn, Alkim, \& Bootsma, 2008) based on the Fulltraffic project dataset (Alkim, Bootsma, \& Hoogendoorn, 2007). The latter studies demonstrated that desired time headway in car-following episodes is contingent upon ACC deactivation and reactivation. Findings from the Fulltraffic project were also used to simulate control transitions from ACC to manual driving in microscopic traffic simulation tools (Klunder, Li, \& Minderhoud, 2009; Xiao, Wang, Schakel, \& van Arem, 2018). Simulation results suggested that transitions can reduce throughput and disrupt traffic flow performance.

However, the most detrimental effects of control transitions are expected to occur if vehicles of higher automation enter public roads. Operators of these vehicles will not be required to monitor the primary driving task. Thus, increased driver distraction prior to a TOR issued by the vehicle automation might ensue reduced driver performance after the ToC, or even an MRM if the ToC is unsuccessful (i.e., the driver fails to respond to the TOR). Nonetheless, there is limited information publicly available with respect to control transitions pertinent to vehicles of higher automation levels. Most relevant studies address human factors and ergonomics aspects of the transitions either with the use of driving simulators or based on real world experiments (Eriksson \& Stanton, 2017; Gold, Damböck, Lorenz, \& Bengler, 2013; Gold, Körber, Lechner, \& Bengler, 2016; Lu, Coster, \& de Winter, 2017; Merat, Jamson, Lai, Daly, \& Carsten, 2014)

. These studies set the ground for the development of a novel ToC model, which is introduced in this paper that can replicate driver behavior during a ToC and vehicle motion during a MRM. The ToC model is integrated into the Simulation of Urban Mobility (SUMO) microscopic traffic simulator (Lopez et al., 2018) to facilitate the simulative assessment of ToCs/MRMs with respect to safety, traffic efficiency and the environment.

Additionally, this paper examines traffic management measures that were developed in the context of the TransAID project (Wijbenga et al., 2018) to mitigate the adverse effects of ToCs/MRMs in 
areas of the road network where traffic operations favor the occurrence of ToCs/MRMs (i.e. work zones, merging areas, lane drops, no automation zones etc.). These areas are referred as "Transition Areas" in the context of this study. The measures presented in this paper were crafted to either prevent ToCs/MRMs by providing vehicle path information or distribute ToCs/MRMs by scheduling their occurrence in space and time assuming that vehicle connectivity (V2X) is available. These measures are investigated with the use of the microscopic traffic simulation tool SUMO. Simulation experiments encompassing driver models both for manual and automated vehicles (including the proposed ToC model) are run with and without the devised traffic management measures. The efficacy of the measures is assessed in terms of generated safety, traffic efficiency and environmental benefits.

The modelling and simulation of manual and automated driving in SUMO is presented in Chapter 2. The traffic management services that were developed by the TransAID project to alleviate the impacts of ToCs/MRMs are described in Chapter 3. Simulation experiments and corresponding results pertaining to each service are discussed and presented in Chapter 4. Finally, this study is concluded in Chapter 0 where outlooks for future work are also introduced.

\section{Modelling}

For the modelling and simulation of traffic scenarios with control transitions from automated to manual driving we have employed the microscopic traffic simulation suite SUMO (Lopez et al., 2018). A major benefit of SUMO is that it is open source, which renders our research transparent and reproducible. The code for the car-following models, the driver state and the ToC device, and the gap controller, which are discussed below, has been made available from the SUMO repository ${ }^{1}$.

\subsection{Car following model for automated vehicles}

In this work, we adopted the ACC car-following model developed in (Liu, Kan, Shladover, Lu, \& Ferlis, 2018; V. Milanés et al., 2014; Vicente Milanés \& Shladover, 2014a; Xiao, Wang, \& van Arem, 2017), wherein the proposed ACC control algorithm is comprised of the following three subcontrollers: a) speed (or cruising) controller, b) gap-closing controller, and c) gap controller. A fourth sub-controller, namely collision avoidance controller, is also introduced in order to prevent rear-end collisions when safety-critical conditions arise. In what follows, the basic definitions and equations corresponding to each control mode (sub-controller) are presented.

\section{Speed Control Mode}

The control objective of the speed control mode is to maintain the pre-defined desired driving speed by the driver and is activated when the preceding vehicle is absent or beyond the on-board sensors' detection range (120 meters in this study). In particular, this mode aims to eliminate the deviation between the vehicle speed and the desired speed and is given in Eqn. (1):

$$
\alpha_{i, k+1}=k_{1}\left(v_{d}-v_{i, k}\right), \quad k_{1}>0,
$$

in which $\alpha_{i, k+1}\left(\mathrm{~m} / \mathrm{s}^{2}\right)$ is the acceleration recommended by the ACC controller to the $i-t h$ subject vehicle for the next time step $k+1 ; v_{d}(\mathrm{~m} / \mathrm{s})$ and $v_{i, k}(\mathrm{~m} / \mathrm{s})$ indicate the desired free flow speed and the current speed of the subject vehicle respectively; $k_{1}$ is the control gain in the speed difference

\footnotetext{
${ }^{1}$ www.github.com/eclipse/sumo
} 
between the free flow speed and the subject vehicle's current speed. Typical values for this gain range between $0.3-0.4 s^{-1}$ according to (Xiao et al., 2017); in this study $k_{1}=0.4 s^{-1}$.

\section{Gap Control Mode}

When the spacing between the subject and the preceding vehicle is smaller than a pre-specified minimum threshold (100 meters in this study), and the gap and speed deviations are concurrently smaller than $0.2 \mathrm{~m}$ and $0.1 \mathrm{~m} / \mathrm{s}$ respectively, the ACC controller activates the gap control mode to enable the subject vehicle to follow the preceding vehicle's motion. This mode is described by the following second-order transfer function based on the gap and speed deviations with respect to the preceding vehicle:

$$
\alpha_{i, k+1}=k_{2} e_{i, k}+k_{3}\left(v_{i-1, k}-v_{i, k}\right), \quad k_{2}, k_{3}>0,
$$

where $e_{i, k}(m)$ represents the gap deviation of the $i$-th subject vehicle at the current time step $k$, and $v_{i-1, k}(m / s)$ is the current speed of the preceding vehicle (index $i-1$ refers to the leader of vehicle $i) ; k_{2}$ and $k_{3}$ are the control gains on both the spacing and speed deviations, respectively. The proposed optimal values for the gains are $k_{2}=0.23 \mathrm{~s}^{-2}$ and $k_{3}=0.07 \mathrm{~s}^{-1}$ (Xiao et al., 2017).

The gap deviation of the $i$-th subject vehicle $\left(e_{i, k}(m)\right)$ is defined in Eqn. (3):

$$
e_{i, k}=x_{i-1, k}-x_{i, k}-t_{d} v_{i, k}
$$

where,$x_{i-1, k}(m)$ is the current position of the preceding vehicle, $x_{i, k}(m)$ is the current position of the subject vehicle, $v_{i, k}(\mathrm{~m} / \mathrm{s})$ is the current speed of the subject vehicle and $t_{d}(\mathrm{~s})$ is the desired time gap of the ACC controller.

\section{Gap-closing Control Mode}

The gap-closing controller enables the smooth transition from speed control mode to gap control mode and is triggered when the spacing to the preceding vehicle is shorter than $100 \mathrm{~m}$. This mode was derived by reducing the gain related to the gap deviation and increasing the gain related to the speed deviation of the gap mode (Xiao et al., 2017). Hence, the control gains of Eqn. (2) are set equal to $k_{2}=0.04 \mathrm{~s}^{-1}$ and $k_{3}=0.8 \mathrm{~s}^{-1}$ in this sub-controller. If the spacing is between the minimum and maximum threshold, the subject ACC vehicle retains the previous control strategy during the previous time step (either speed or gap-closing control mode) to provide hysteresis in the control loop and perform a smooth transfer between the two strategies.

\section{Collision Avoidance Control Mode}

This study introduced the collision avoidance control mode to prevent rear-end collisions occurring during simulations due to safety critical conditions (i.e. low time-to-collision (TTC) values, follower's speed significantly higher than the leader's). The collision avoidance mode was also derived by tuning the control gains of the existing gap mode and is triggered when the spacing to the preceding vehicle is smaller than $100 \mathrm{~m}$ and concurrently the gap deviation is negative. In this case, the control gains of Eqn. (2) are set as $k_{2}=0.8 \mathrm{~s}^{-2}$ and $k_{3}=0.23 \mathrm{~s}^{-1}$ to ensure that ACC vehicles can brake hard enough to avoid an imminent collision 


\subsection{Car following model for manually controlled vehicles}

In the following we describe the extensions we have incorporated into SUMO to model manually controlled vehicles. The main difference we take into account when differentiating manual from automated driving is the imperfection of the human driver. Indeed, a number of studies report that for some time after the transition of control the driver performance is decreased, which seems to be correlated to a reduced awareness of the traffic situation while the driver is redirecting his or her focus to the driving task (Fuller, 2005; Lu et al., 2017; Merat et al., 2014; Young \& Stanton, 2002).

Characteristic measures of performance or involvement regarding the driving task that possess a correlate in a microscopic simulation are for instance:

- reaction time,

- lane keeping,

- headway fluctuations,

- lane change reluctance, or

- braking intensity.

To reproduce these phenomena of reduced driver performance, we have developed and implemented a model for the driver's state into SUMO. It is available as a vehicle device ${ }^{2}$ since SUMO version 1.0. Besides lateral inaccuracies, i.e., lane keeping, it affects all of the above points in a direct or indirect way.

In the simulation study presented in Sections 3 and 4 we employ SUMO's standard car following model as a basis for manually controlled vehicles (MV). It is a variant of the Krauss model (Krauß, 1998; Krauß, Wagner, \& Gawron, 1997; Lopez et al., 2018), which , in turn, is based on the same principle as the Gipps car following model (Gipps, 1981; Wilson, 2001). That is maintenance of a safe following speed at all times, expressed by the equation

$$
\underbrace{\frac{\left(v_{f}+\tau \cdot a\right)^{2}}{2 b_{f}}}_{\begin{array}{c}
\text { braking distance } \\
\text { of follower }
\end{array}}+\underbrace{v_{f} \cdot \tau}_{\begin{array}{c}
\text { reaction distance } \\
\text { of follower }
\end{array}} \leq \underbrace{\frac{v_{l}^{2}}{2 b_{l}}}_{\begin{array}{c}
\text { braking distance } \\
\text { of leader }
\end{array}}+\underbrace{\Delta x}_{\begin{array}{c}
\text { current } \\
\text { headway }
\end{array}},
$$

which defines a desired acceleration $a=f\left(v_{f}, \Delta v, \Delta x\right)$ for the follower, where $\Delta v=v_{l}-v_{f}$, by choosing $a$ as the maximal value fulfilling (4). Note that some additional provisions are made for the case $b_{l}<b_{f}$, see (Lücken, 2019). Here, $v_{f}$ and $v_{l}$ are the current velocities of the follower and the leader, respectively, $b_{f}$ and $b_{l}$ are the corresponding assumed braking rates, $\tau$ is interpreted as the desired reaction time buffer for the follower and equals to the stationary temporal headway in equilibrium flow. Since the model is based on the principle of safety, it is a popular choice for a simulative evaluation of traffic safety related aspects (Saifuzzaman, Zheng, Haque, \& Washington, 2015; Xin, Hourdos, Michalopoulos, \& Davis, 2008).In the following we give the technical details for the driver state model. The dynamical core component is a stochastic process $\mathrm{H}$, which models the amplitude of the driver's errors. Its dynamics are given as

$$
d \mathrm{H}_{t}=-\theta_{t} \cdot \mathrm{H}_{t} \cdot d t+\sigma_{t} \cdot d W_{t}
$$

Note that for a fixed timescale $\theta_{t} \equiv \theta_{0}$ and fixed intensity $\sigma_{t} \equiv \sigma_{0}$, this is an Ornstein-Uhlenbeck process (Gardiner, 2009; Kesting \& Treiber, 2013). However, in the proposed model, $\theta$ and $\sigma$ are

\footnotetext{
${ }^{2}$ https://sumo.dlr.de/wiki/Driver_State
} 
considered non-constant to reflect the variability of the driver's awareness over time. Stipulating a time variant quantity $A(t) \in[0,1]$, called awareness in the following, we assume that

$$
\theta_{t}=c_{\theta} \cdot A(t) \text { and } \sigma_{t}=c_{\sigma} \cdot(1-A(t)) .
$$

with scaling coefficients $c_{\theta}$ and $c_{\sigma}$. Roughly speaking, this implies that the higher the awareness, the faster the errors decay and the smaller is their range.

As a general mechanism applicable to a wide range of car-following models, we propose to impose the generated errors at the level of perception as described in the following. Given undisturbed car-following dynamics of the form

$$
a=f\left(v_{f}, \Delta v, \Delta x\right),
$$

we model the dynamics under reduced driving performance as

$$
a=f\left(v_{f}, \Delta \tilde{v}, \Delta \tilde{x}\right),
$$

where

$$
\Delta \tilde{x}=\Delta x+\eta_{x} \text { and } \Delta \tilde{v}=v+\eta_{v}
$$

are the perceived spacing and the perceived speed difference with effective spacing error $\eta_{x}$ and effective speed difference error $\eta_{v}$. These effective errors are assumed to be proportional to the distance $\Delta x$ to the leading vehicle (Xin et al., 2008) and the main error term $\mathrm{H}_{t}$, that is,

$$
\eta_{x}(t)=c_{x} \cdot \Delta x(t) \cdot \mathrm{H}_{t} \text { and } \eta_{v}(t)=c_{v} \cdot \Delta x(t) \cdot \mathrm{H}_{t},
$$

with constant coefficients $c_{x}$ and $c_{v}$.

As an additional, generic mechanism for imperfect driving, perception specific action points are taken into account (Todosiev, 1963; Xin et al., 2008). An action point is a time point $t$ where the acceleration $a(t)$ is changing its value according to the dynamical equation of the given car-following model, i.e. Eqn. (8).

Further, it is assumed that a change in a perceived quantity is only recognized if its magnitude surpasses a certain threshold value. Accordingly a corresponding change in action, here, a change of acceleration, is only taken out when the currently perceived speed difference $\Delta \tilde{v}(t)$ deviates sufficiently from the last recognized value $\Delta \tilde{v}_{\text {rec }}$ or the currently perceived gap $\Delta \tilde{x}(t)$ deviates from the value estimated based on the last recognized quantities. That is, time $t$ is assumed an action point if either

$$
\left|\Delta \tilde{x}_{\text {rec }}+\left(t-t_{\text {rec }}\right) \cdot \Delta \tilde{v}_{\text {rec }}-\Delta \tilde{x}(t)\right|>\theta_{x} \text {, or }\left|\Delta \tilde{v}_{\text {rec }}-\Delta \tilde{v}(t)\right|>\theta_{v} .
$$

If not mentioned explicitly, we use the following values for the parameters of the driver state model:

$$
c_{\theta}=100, c_{\sigma}=0.2, c_{v}=0.15, c_{x}=0.75, \theta_{v}=0.1, \theta_{x}=0.1
$$

These are also the default values assigned on device initialization by SUMO.

In Figure 1 we show an example for a trajectory generated by a Krauss car-following model equipped with a driver state device, i.e., with superimposed dynamic perception errors and a constant awareness level $A(t) \equiv 0.1$.The Krauss vehicle was inserted just behind a leading vehicle, whose speed was controlled via TraCI to match an empirically measured trajectory, see black line in Figure 1(a). The empirical trajectory of the true follower vehicle is shown in green color for the purpose of qualitative 
comparison with the model trajectory (magenta). The underlying driving episode of approximately three minutes duration was extracted from the $\operatorname{sim}^{\mathrm{TD}}$ database $\mathrm{e}^{3}$. The experimental parametrization for the driving error dynamics was obtained from a manual fit of the mean headway (Figure 1(c)) and the region covered by the trajectory in the $(\Delta x, \Delta v)$-plane (Figure $1(\mathrm{~d}))$. This preliminary calibration led to qualitatively matching characteristics of the empirical and model trajectory in the respective dimensions (panels (c) and (d)). However, panels (a) and (b) of Figure 1 suggest that further work is required to achieve a more realistic behavior, especially regarding amplitude and frequency of speed oscillations, i.e., the acceleration behavior.

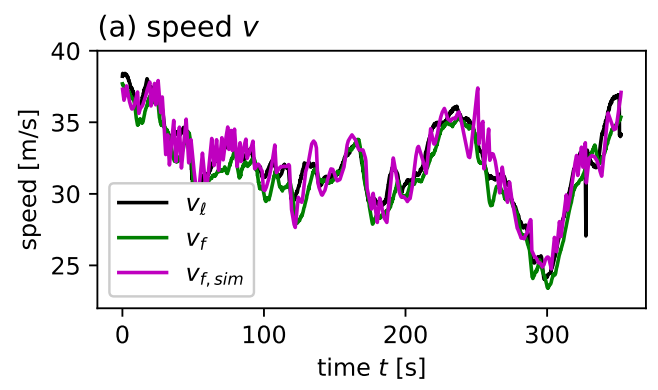

(c) time headway [s]

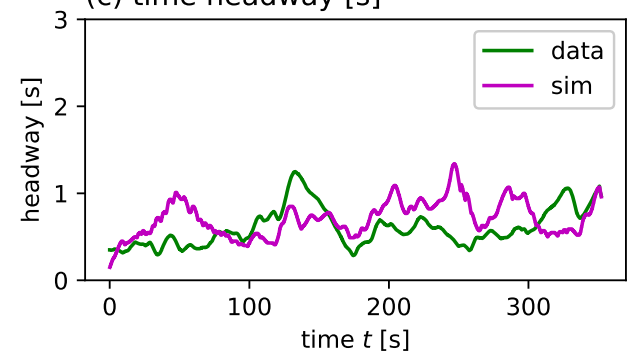

(b) speed difference $\Delta v$

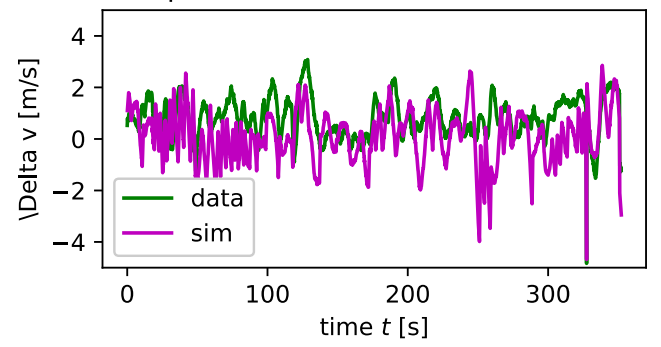

(d) time headway vs. $\Delta v$

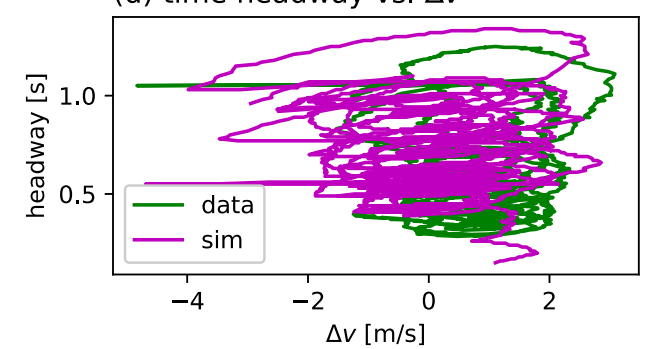

Figure 1: Car-following trajectories for an episode extracted from empirical data $\left[\right.$ sim $^{\mathrm{TD}}$ database $^{3}$ ] (green line), and generated by a Krauss car-following model with superimposed driver-state device at constant awareness level $A(t) \equiv 0.1$ (magenta line). The black trajectory in (a) is the empirically measured speed of the leading vehicle.

\subsection{SUMO's ToC model}

The modelling of vehicles with changing control regimes, i.e. manual and automated driving modes, has to incorporate a mechanism to switch between these. This ability of dynamically choosing different parameter sets for, e.g., the underlying car-following or lane changing models, or even exchanging one model for a different one, is a minimal requirement for such a model. However, for the purpose of representing control transitions it seems desirable to include elements that describe the processes surrounding the takeover. The complete model for depicting control transitions in SUMO has been incorporated into a vehicle device ${ }^{4}$. Besides managing the switching between different models for automated and manual driving, it optionally controls the processes during $\mathrm{ToC}$ preparation and a phase of decreased driving performance after a downward transition, i.e., when manual control is resumed, see Figure 2.

\footnotetext{
${ }^{3}$ https://www.sit.fraunhofer.de/de/simtd/

${ }^{4}$ https://sumo.dlr.de/wiki/ToC Device
} 


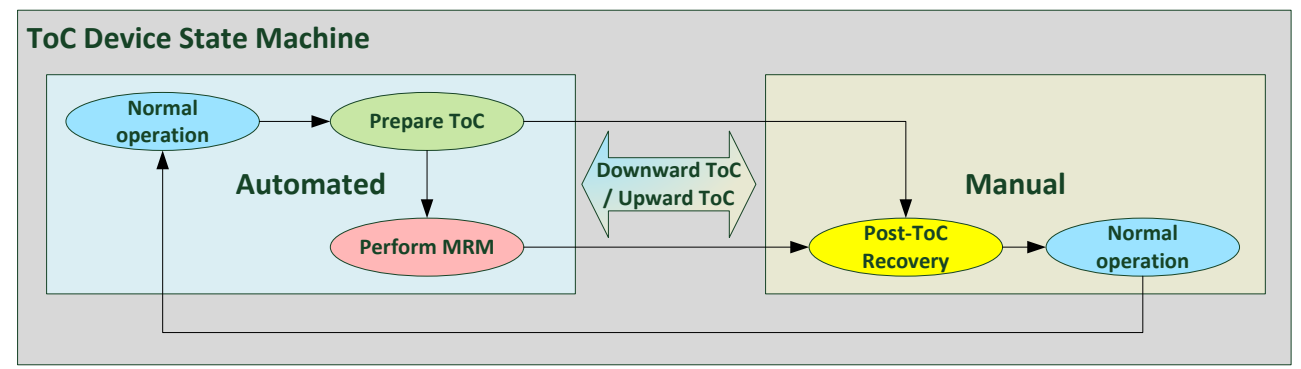

Figure 2: State transition diagram for SUMO's ToC model. The main operational modes (Automated and Manual) each possess various submodes associated to processes during a takeover.

Firstly, we have incorporated a gap control mechanism into SUMO, which allows the user to impose a continuous adaptation of a vehicle's desired headway. On one hand this mechanism may be employed to smoothly adapt the desired headway of the regime, which is currently operational, towards the new value of the regime targeted by the transition. In particular, when switching discontinuously from a small towards a large desired headway smoothing the transition may prevent artificially high braking resulting from the discontinuity. Moreover, we employ the gap control to depict a preparatory phase in case of a downward transition (from automated to manual), where it seems reasonable to assume that the vehicle automation may enlarge the headway to the leading vehicle in order to simplify the takeover situation for the human driver. The gap control mechanism can be either triggered automatically by an appropriate configuration of the ToC model (as described below), or directly via SUMO's online interface $\mathrm{TraCI}^{5}$.

The user can configure the following aspects of the process:

a) the new desired time, which acts on the car-following model's parameter tau,

b) the new desired spacing, which is incorporated in an additive fashion (i.e., the effective value is subtracted from the space gap argument $\Delta x$ of the car-following model),

c) the duration, for which the control stays active after the desired gap has been established,

d) the linear rate, at which the old headways are adapted towards the given values,

e) an upper bound for the braking rate employed to comply with the imposed headway (optional),

f) a fixed reference vehicle to which the headway should be established (optional).

The functionality (f) is not needed for the objectives of modeling control transitions, but was included with applications for cooperative maneuvering in mind. Automated triggering of the gap control simultaneously to a TOR can be added to the ToC model by setting at least one of the corresponding device parameters (prefixed with $\circ$ g...).

Further, to incorporate the decreased driving performance after a downward transition, i.e., after the driver resumes the vehicle control, the ToC device allows specifying the level of awareness $A_{0}$ at which the driver resumes control, as well as the linear rate, at which the awareness recovers to the full value $A=1.0$, i.e. normal performance (see Section 2.2 and Figure 3).

The $\mathrm{ToC}$ model adds an online interface, which allows triggering control transitions between specified models for automated and manual driving modes ${ }^{6}$. Such a TOR specifies the lead time available for the driver to take back the control. If the driver does not act in time, a MRM will be initiated, bringing the vehicle to a full stop at a constant deceleration rate, if the driver does not intervene in the meantime, cf. Figure 3.

\footnotetext{
${ }^{5}$ https://sumo.dlr.de/wiki/TraCI/Change Vehicle State\#open gap .280x16.29

${ }_{6}^{6}$ https://sumo.dlr.de/daily/pydoc/traci._vehicle.html\#VehicleDomain-requestToC
} 


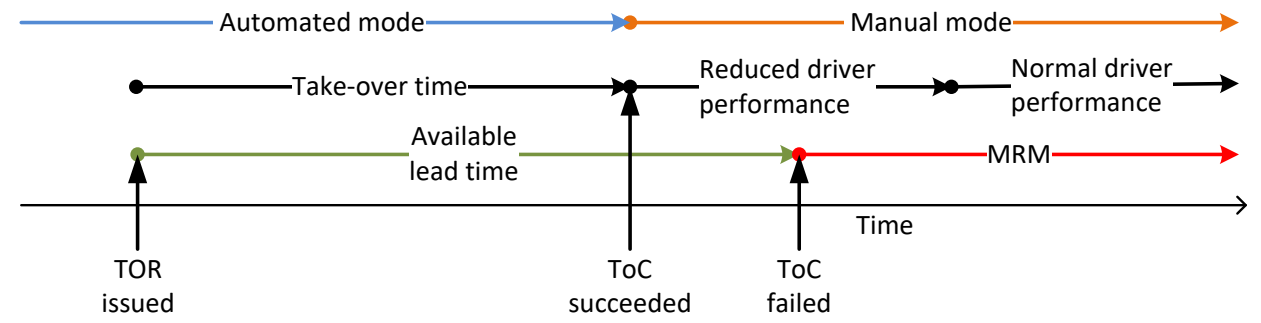

Figure 3: Timelines for a successful and a failing takeover.

To configure a ToC device for a vehicle the user has to specify the SUMO vehicle types for the manual and automated regime. Other, optional parameters, which take default values if not specified by the user, may be given to control the driver's response time, the time the driver abstains from lane changes after the takeover, and the deceleration rate applied during a minimum risk maneuver. Further, the temporal characteristics of the post-ToC phase of reduced driver performance may be controlled by two parameters: the driver's initial awareness $A_{0}$, and the corresponding awareness recovery rate $r$, giving for the post-ToC awareness:

$$
A\left(t_{0}+t\right)=\min \left(1.0, A_{0}+r t\right)
$$

which affects the driver state dynamics as described in Section 2.2.

\section{Simulation of TransAID Services}

Using different modeling schemes (pessimistic and optimistic with respect to safety and efficiency) and scenario parameters (traffic demand and share of automated vehicles), we have simulated different scenarios of transition areas, i.e. road sections where an accumulated occurrence of control transitions may occur (Evangelos Mintsis et al., 2018; Maerivoet et al., 2019). These scenarios were intended as use cases for the application of specific traffic management services devised in the framework of the TransAID project. For each scenario, we have compared the values of several indicators regarding traffic safety, efficiency, and emissions in presence and absence of the traffic management measures. In the following we report on the results for two of these use cases.

\subsection{Scenario 1: Providing path information}

In some situations AVs may not be able to trespass an obstacle, where an advised circumvention is not conceived by the automation. In these cases the proportion of vehicles, which need to issue a ToC, may be reduced significantly if according path information informing the vehicle of an alternative trajectory for passing the obstacle is provided via a road side unit (RSU). This information is assumed to take the form of an update suggestion for the vehicle's digital map, e.g., as a MAPEM (ETSI, 2016).

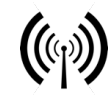




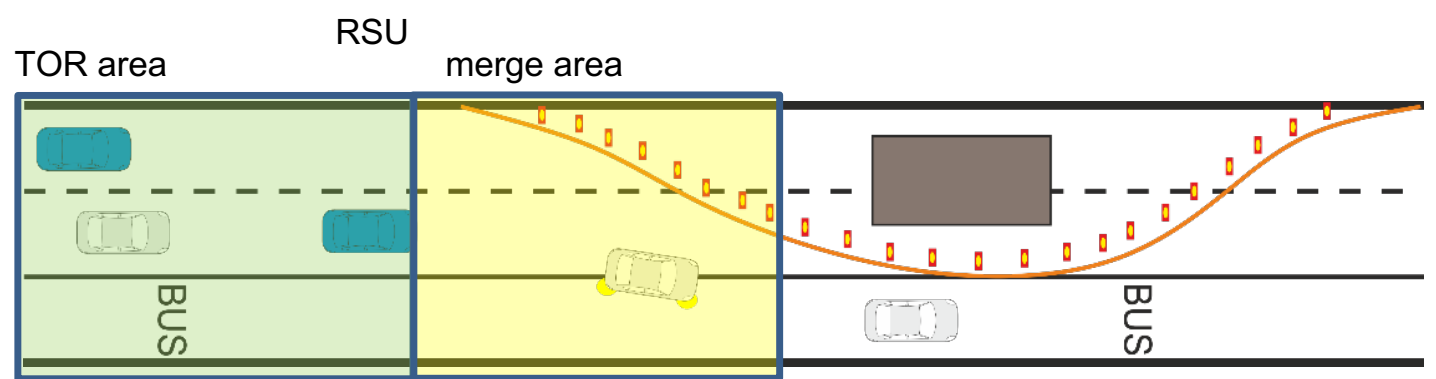

Figure 4: Schematic representation of Scenario 1. AVs and MVs approach an obstacle on a two-lane urban corridor with an extra lane usually reserved for public transport.

We consider a two-lane road section with an additional lane reserved for public transport, where a construction site is installed across both main lanes, see Figure 4. During the time of the road closure, vehicles are permitted to use the public transport lane as indicated by customized road signs. We assume that only a fraction of the AVs in the scenario can process the information of these signs and the others request a control transition in the absence of traffic management measures, i.e. if no further information is provided. For the simulations presented here we assumed that the rate of automated passages would increase from 25 to 75 percent if information about the usability of the bus lane is regularly broadcasted to all AVs. That means in the scenario without traffic management, we assumed that 75 percent of all AVs undertake a downwards transition, and that in presence of traffic management this rate would drop to 25 percent.

Besides the additional path information provided, the TM addresses individual AVs and recommends enlarging headways in the merging area before the lane reduction to facilitate merging of vehicles on adjacent, ending lanes.

The roadside infrastructure (RSI) monitors the approaching vehicles, especially within the merging area (see Figure 4), and regularly broadcasts the information regarding the alternative path around the construction side, i.e. the release of the lane restrictions for the rightmost lane. For vehicles on the rightmost lane, that are located within the merging area, the RSI sends an individual advise to increase the headway to their leading vehicles if other vehicles are present on the other lanes within the merging area. AVs that receive a headway suggestion are assumed to comply with that suggestion, and AVs that receive the path information will incorporate it into their trajectory planning.

To model the reception of the path information for a AV, that is the update of its digital map to incorporate the permission to drive on the rightmost lane, we used SUMO lane permissions in combination with manipulating the vehicle class of the corresponding SUMO vehicle. That is, the

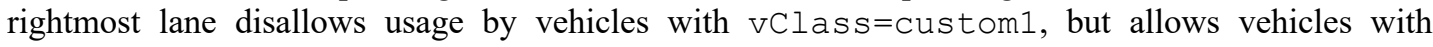
$\mathrm{vClass}=$ custom 2 . Thus, we initiate the vehicle type of AVs to be of class custom 1 at insertion and set the vehicle class to custom2 at reception of the map information from the RSU.

\subsection{Scenario 2: Broaden the distribution of takeover requests}

In the second scenario considered, a two-lane motorway, we assume the existence of a road section where automated driving is not possible (No-AD zone), e.g., due to adverse road conditions or legal regulation. Without further assumptions one may expect that automated vehicles, which approach the No-AD zone, request a takeover as close as feasible to the No-AD zone. An approximate distance for the latest point to request a takeover with a given lead time $T_{\text {lead }}$, anticipated speed $v_{0}$ and assuming a constant braking rate $b_{\text {MRM }}$ during an eventual minimum risk maneuver, can be estimated as 


$$
d_{\text {min }}=\underbrace{T_{\text {lead }} \cdot v_{0}}_{\begin{array}{c}
\text { distance travelled } \\
\text { until MRM }
\end{array}}+\underbrace{\frac{v_{0}^{2}}{2 b_{\mathrm{MRM}}}}_{\begin{array}{c}
\text { stopping distance } \\
\text { in case of MRM }
\end{array}} .
$$

To ensure that only manually controlled vehicles enter the No-AD zone, an automated vehicle should request a takeover at least at a distance $d_{\min }$ before the begin of the No-AD zone.

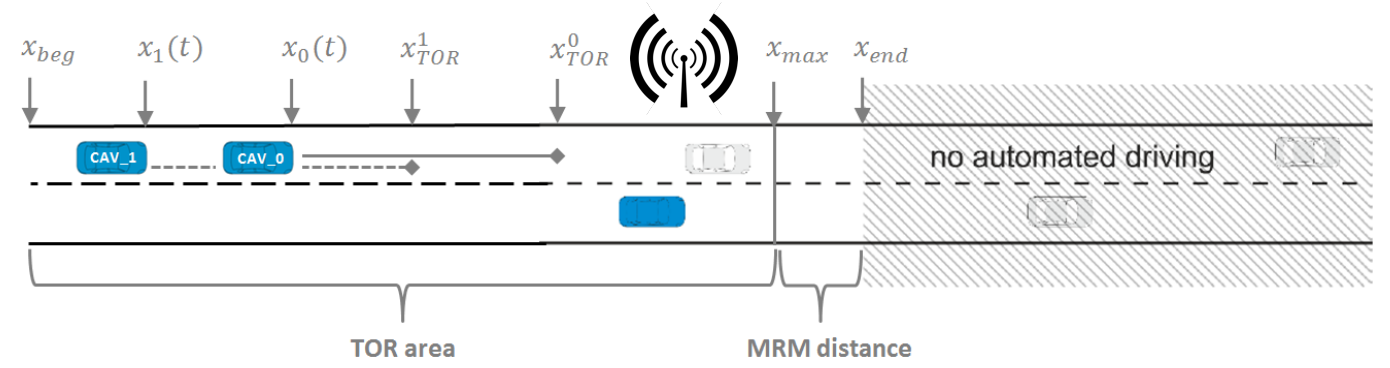

Figure 5: Schematic representation of Scenario 2.

Figure 5 shows the schematic representation of the scenario and locations of significance for the control algorithm. The area, wherein AVs are expected to receive TORs and perform ToCs stretches from position $x_{b e g}$ to $x_{\text {max }}$, denoting the location where either a ToC must have been taken out, or an MRM must be initialized latest to assure that only manually driven vehicles enter the No-AD zone beginning at $x_{\text {end }}=x_{\max }+v_{\max }^{2} / 2 b_{\mathrm{MRM}}$. The depicted situation shows two AVs, CAV_O and $C A V_{-}$, with current position $x_{1}(t)$ and $x_{2}(t)$, respectively, approaching the No-AD zone. The TMC has already scheduled TOR positions for these vehicles at $x_{T O R}^{0}$ and $x_{T O R}^{1}$. When the TMC recognizes that a vehicle passes its assigned TOR position, a TOR is sent to this vehicle.

The RSI monitors the position and speed of the approaching vehicles and the total traffic density within the TOR area, cf. Figure 5. As the sequential scheduling of TORs for strings of AVs is an essential component for the distribution algorithm (see also Figure 6) the TMC organizes AVs entering the TOR area in such groups and assigns TOR positions for all AVs of a group when it is finalized. The finalization of a group is taken out if no more vehicles will be added since the distance of the last vehicle to the TOR area entry at $x_{\text {beg }}$ exceeds a threshold or an MV is trailing the last vehicle. Further, the TMC updates the assigned TOR positions according to the current vehicle speeds in every control step as described in detail in (Maerivoet et al., 2019). AVs regularly broadcast their current state (position and speeds, e.g. via CAM messages), wait for TORs from the RSU, and initiate the $\mathrm{ToC}$ at reception.

Our simulations show that under some circumstances, it can be favorable to issue a part of the TORs earlier to prevent the accumulation and summation of possible traffic perturbations related to the takeover processes (see Section 4.2), especially if the takeover process involves a, possibly only temporary, increased headway at some point in time. To illustrate this we assumed that a AV begins to smoothly (braking with $\leq 1.0 \mathrm{~m} / \mathrm{s}^{2}$ ) establish a gap of 3.5 seconds after it has firstly communicated a TOR to the driver. 

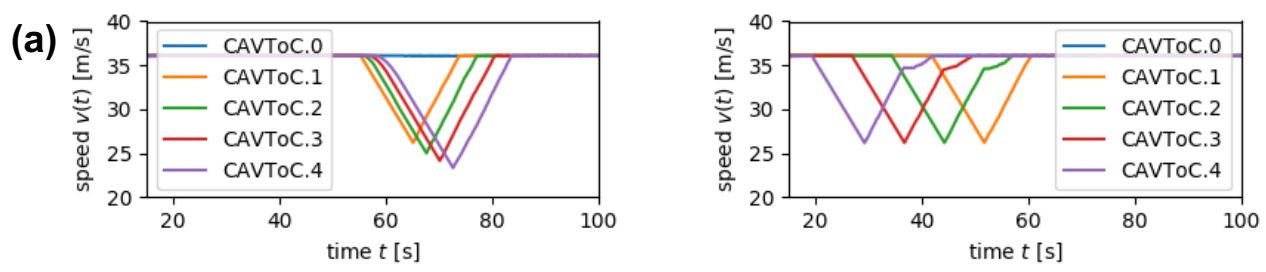

Figure 6: Numerical experiments for collective disengagement in a string of automated vehicles with preparatory headway increment. (a) Quasi-synchronous disengagement at a specific location; (b) managed sequential disengagement.

To demonstrate the possible benefits of distributing takeovers in this case we use a simple distribution algorithm based on the vehicle density on the road section approaching the No-AD zone, which could in reality be made available for the traffic management by traffic detectors, e.g. cameras or induction loops, on that section. TORs are directed to the approaching AVs the earlier if the observed density is high, since the density can be used as an indicator of present disruptions within the approaching section. More importantly, within groups of AVs we apply a sequential scheduling of TORs preventing a collective transition that would add up to a larger perturbation of the flow, see Figure 6.

\section{Results}

To evaluate the impact of the proposed TM measures we have taken out simulations covering a broad range of parameter combinations, where we varied the traffic demand level, the share of $\mathrm{C}(\mathrm{A}) \mathrm{Vs}$ in the vehicle fleet mix, and the parametrization scheme for the vehicle models regarding traffic safety and efficiency (Maerivoet et al., 2019). For each combination we executed ten simulation runs, each of one hour simulated time, see the Appendix for the model parametrization. Here we show results for a moderate choice of model parameters (see Appendix) and traffic demand level C (i.e. $77 \%$ of the assumed capacity), which results in 1155 vehicles/hour for Scenario 1 and 3234 vehicles/hour for Scenario 2.

Simulation experiments encompass the following different vehicle types: a) manually driven vehicles (MVs), b) connected vehicles (CVs), and c) connected and automated vehicles (CAVs). The motion of each vehicle type in the SUMO simulation environment is dictated by the models presented in Table 1. CVs are assumed to be only longitudinally automated, while AVs can both throttle/brake and steer during automated driving mode. Moreover, ToCs are considered instantaneous for CVs (driver is in the loop and instantly resumes vehicle control), while AV drivers are allowed to be involved in secondary tasks and thus might not respond in a timely fashion to the TOR. Both vehicle types (CVs and CAVs) have communication capabilities with the infrastructure (V2I). 
Table 1: SUMO models emulating the motion of the considered vehicle types

\begin{tabular}{lccc}
\hline SUMO Model & MV & Vehicle Type & CAV \\
\hline Krauss CF Model & $\checkmark$ & CV & $\times$ \\
ACC CF Model & $\times$ & $\checkmark$ & $\checkmark$ \\
Default LC Model $^{7}$ & $\checkmark$ & $\checkmark$ & $\times$ \\
Parametrized LC $_{\text {Model }^{8}}$ & $\times$ & $\times$ & $\checkmark$ \\
ToC Model & $\times$ & $\checkmark$ & $\checkmark$ \\
\hline
\end{tabular}

The traffic mix was varied across three different penetration rates of automated vehicles resembling the shares in the following Table 2.

Table 2: Simulated traffic mixes

\begin{tabular}{lccc}
\hline & MV & CV & CAV \\
\hline Mix 1 & $70 \%$ & $15 \%$ & $15 \%$ \\
Mix 2 & $50 \%$ & $25 \%$ & $25 \%$ \\
Mix 3 & $20 \%$ & $40 \%$ & $40 \%$ \\
\hline
\end{tabular}

\subsection{Scenario 1: Providing path information}

In Scenario 1 we examined the impact of informing CAVs of an alternative pathway around an upcoming obstacle, thereby preventing the occurrence of ToCs for more AVs than in the case of not providing any information. Figure 7 shows a snapshot of the merge area in the corresponding simulation scenario. One may observe a CAV merging in front of another CAV, which increased its headway to the leading MV in order to facilitate the merging. The presence of automated vehicles on the rightmost lane indicates that they incorporated the suggestion to pass the obstacle using the reserved lane.

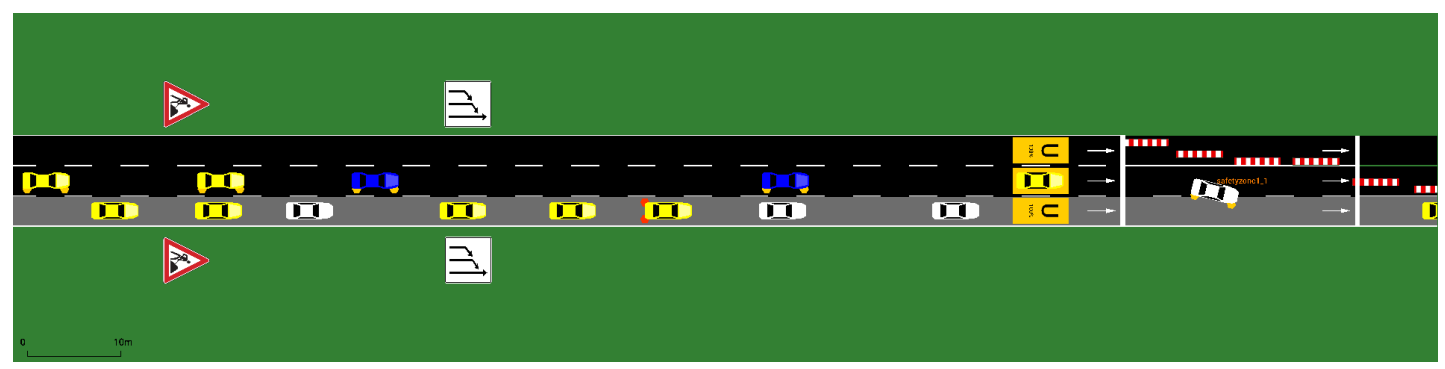

Figure 7: Detail view of the merging area in the SUMO scenario. The grey lane is usually reserved for public transport but opened temporarily to provide a possibility to pass the construction works stretching over the two main lanes. Vehicle colors indicate the vehicle type: yellow - MV; blue-CAV; white - CV.

Preliminary results for scenario 1 show that the main effects of the proposed TM measures seem to regard traffic safety while it has little effect on traffic efficiency and emissions (see Figure 8). As an indicator for safety we used the number of episodes where the TTC of two vehicles in a lead-follow

\footnotetext{
${ }^{7}$ The default SUMO lane change model is thoroughly presented in (Erdmann, 2014).

${ }^{8}$ The parametrized lane change model that reflects CAV lane change behavior is described in (Evangelos Mintsis et al., 2018).
} 
relation undercuts a critical threshold of 3 seconds, see Figure $8(\mathrm{~b})$. For the definition and implementation of the TTC, we refer to (Gettman \& Head, 2003) and the SUMO wiki ${ }^{9}$, respectively. The number of critical events dropped significantly when traffic management measures were introduced in the scenario, which can be attributed to the decreased number of ToCs, which also reduces delayed reactions to decreasing headways.

We also hypothesize that the increased headways assumed for CAVs in general but specifically in the merging section contribute to this safety improvement. Differentiating between the effect originating from driving errors during the post-ToC phase and headway effects would require the classification of TTC episodes with respect to the interacting vehicles' automation states, which is work to be done at the day of writing. Especially in view of the increased average headways in the presence of traffic management measures it is remarkable that the traffic efficiency (Figure 8(a)) is not affected by the increased number of vehicles traveling in automated mode.

Figure 8(c) shows the $\mathrm{CO}_{2}$ emissions per kilometer traveled. Similar as for the throughput, no large impact can be observed here. The amount of emitted $\mathrm{CO}_{2}$ was calculated using SUMO's PHEMlight module ${ }^{10}$.
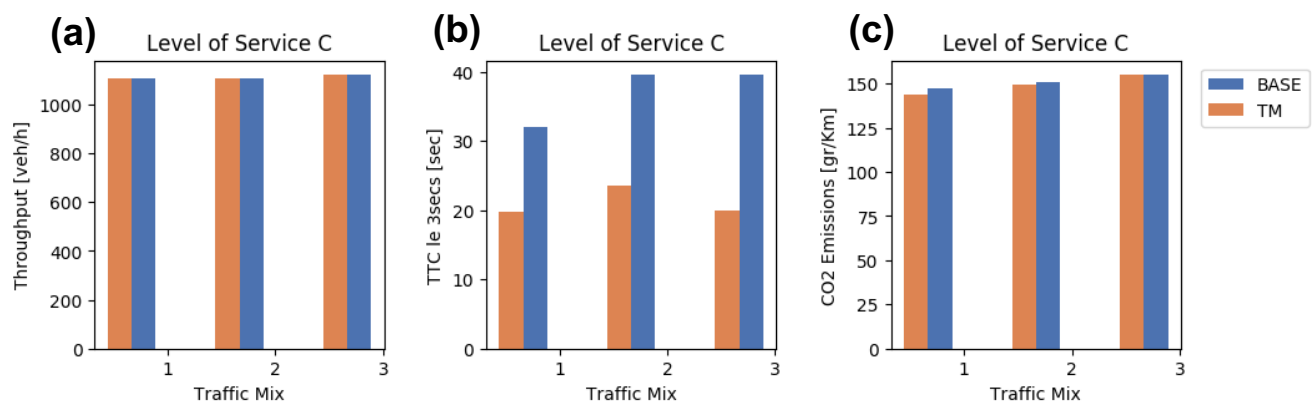

Figure 8: Comparison of cumulative measures for Scenario 1 in presence (orange) and absence (blue) of traffic management. (a) Average throughput (arrived vehicles) within the first hour of simulation over all runs; (b) Average number of TTC episodes with TTC value less than three seconds; (c) Average amount of emitted $\mathrm{CO}_{2}$ per kilometer driven, as calculated by SUMO's PHEMlight model.

\subsection{Scenario 2: Broaden the distribution of takeover requests}

This section presents preliminary results for the impact of distributing TORs spatially over an approaching road section upstream of a No-AD zone. Firstly, we observe that the throughput decreases with an increasing share of CAVs in absence as well as in presence of TM, c.f. Figure 9(a). This result is expectable because of the parametrization of CAV models, in particular larger headways and less agile acceleration. However, the results also indicate that the TM measures taken may dampen the capacity drop induced by larger shares of CAVs.

The effects on traffic safety are more pronounced as shown in Figure 9(b). While the number of critical TTC occurrences rises significantly with the number of present CAVs (and hence the number of ToCs), it stays approximately constant in presence of TM measures. This strongly indicates that the sequential scheduling of ToCs as orchestrated by the TMC for a string of CAVs is highly preferable to a decentralized disengagement, which may be expected to occur in a more synchronized fashion at a specific distance to the No-AD zone entry and would lead to higher amplitudes of corresponding traffic perturbations as suggested by the numerical experiments shown in Figure 6.

\footnotetext{
${ }^{9}$ https://sumo.dlr.de/wiki/Simulation/Output/SSM Device

${ }^{10} \mathrm{https}: / /$ sumo.dlr.de/wiki/Models/Emissions/PHEMlight
} 
Larger perturbations do not only induce unsafe situations but also increase speed variations and consequently higher amounts of deceleration and acceleration, which in turn, cause increased emissions per kilometer traveled as illustrated by Figure 9(c).
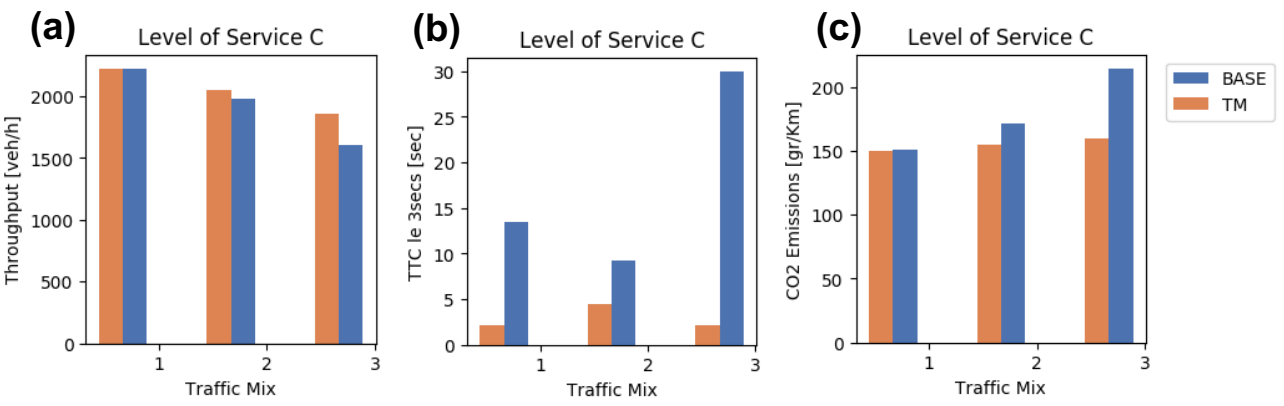

Figure 9: Comparison of cumulative measures for Scenario 2 in presence (orange) and absence (blue) of traffic management. (a) Average throughput (arrived vehicles) within the first hour of simulation over all runs; (b) Average number of TTC episodes with TTC value less than three seconds; (c) Average amount of emitted $\mathrm{CO}_{2}$ per kilometer driven, as calculated by SUMO's PHEMlight model.

Figure 10 shows the spatiotemporal dynamics for a specific simulation run in absence [panels (a), (c)] and presence [panels (b), (d)] of TM measures. In absence of the TMC a perturbation due to simultaneous ToCs of several AVs leads to the development of an extended traffic jam pinned to the entry of the No-AD zone, which does not dissolve until one hour simulation time has elapsed (cf. red area in panel (a)), as opposed to the overall smooth flow preserved in presence of the TMC (cf. panel (b),(d)). For other runs we observed that traffic jams form later and sometimes dissolve during the course of the simulation but the overall result clearly shows that a distribution of TORs may be highly beneficial for a smooth and safe traffic flow.

(a) LOS: C - Traffic Mix: 3 - Seed: 6

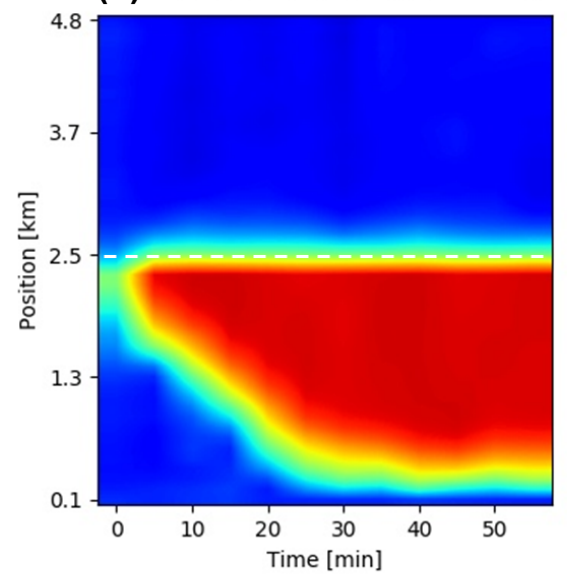

(b) LOS: C - Traffic Mix: 3 - Seed: 6

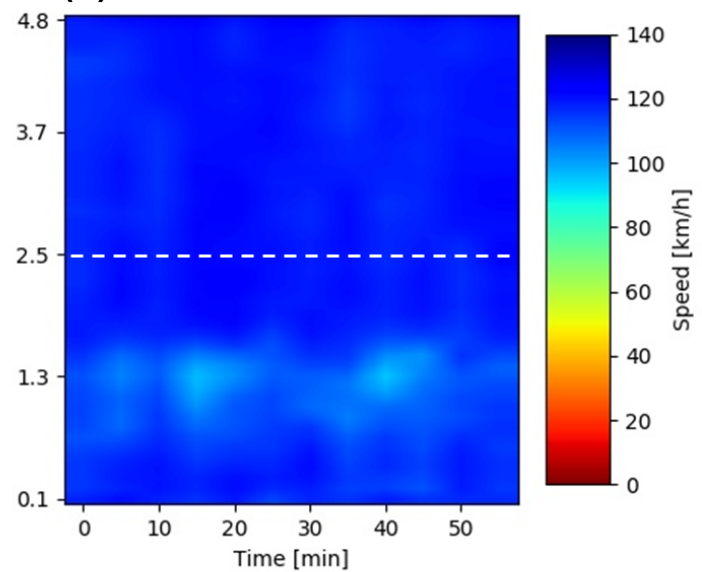

Figure 10: Spatiotemporal diagrams for the mean speed along the simulated road section for an exemplary simulation run. The white, dashed line indicates the entry of the No-AD zone at $\mathrm{km} \mathrm{2.5.} \mathrm{(a)} \mathrm{Formation} \mathrm{of}$ an extended congested area in absence of TM measures; (b) Predominantly smooth flow for a distributed scheduling of TORs. 


\section{Discussion}

In the present paper we have described several extensions, which have been implemented into SUMO in order to reflect mixed traffic conditions, where automated and manual vehicles coexist in one scenario. More specifically, we have implemented an ACC model, which is often applied to model automated controllers (Milanés \& Shladover, 2014; Xiao, Wang, \& van Arem, 2017) and a generic mechanism to impose perception errors upon an arbitrary car-following model. Further, we have presented simulation results for two scenarios where the new models have been applied to evaluate the effects of different TM measures for transition areas, where an increased amount of ToCs can be expected to occur. These results suggest that both cases bear the potential for considerable benefits if the TM is applied. In the simulation the TMC significantly increased either traffic safety (see Section 3.1) or traffic efficiency (see Section 3.2).

Although it is highly probable that the results will hold qualitatively for a wide range of situations as they seem to depend on rather generic properties of the different scenarios (such as the vehicle mix, desired headways, the accepted gaps for lane changes, or the imprecisions of the driver's performance after the takeover), several possible improvements are conceivable regarding the realism and accuracy of the simulations.

Firstly, since the proposed TM measures essentially rely on vehicular communications, it would be important to estimate the reduction of the TM performance caused by error rates and latencies in the message transmission. It seems likely that the amount transmission failures would decrease the degree of the observed improvements induced by the TM by a proportional quantity. However, the foreseen changes may depend non-linearly on the transmission error rate and, moreover, the error rate might relate non-trivially to the traffic density, such that it is a subject worth further studies.

Also, the parameter choices for automated vehicle control are rather speculative since empirical data is not available and may influence the results quantitatively. Nevertheless, as long as the driving behavior of automated vehicles is assumed more conservative than that of MVs, we expect the reported results to persist. In this context, it could be important to include the factor of cooperative maneuvering, which, for instance, might allow automated vehicles to follow each other with highly decreased headways, thereby inverting the assumption of larger headways for CAVs, which we supposed for our simulations so far. It is clear that such a scenario would even raise the profit of reducing ToCs due to a persistent capacity drop associated to the mode switch from automated to manual.

Moreover, the models of human driving may be improved, especially to obtain more reliable results regarding the estimation of traffic safety implications of the evaluated TM procedures. Here, we see potential in refining the error characteristics, which might be achieved by a different calibration of the driver state model, or require additional model components. Our preliminary efforts to achieve a qualitative agreement of the model behavior with a trajectory of a human driver (in a noncritical car-following situation) did not lead to an ultimately satisfactory result (see the discussion of Figure 1). Here, a more careful calibration could take into account the power spectrum or autocorrelation of the speed differences, or distributions of safety surrogate measures as the TTC. In particular, the model's behavior in critical situations has not been tested at all. This is not a straightforward task, though, since corresponding data is very scarce. Possible, additional model components to be considered are situation (e.g. traffic complexity) dependent awareness levels, in particular the mechanism of task difficulty homeostasis (Fuller, 2005; Saifuzzaman et al., 2015). Also, lateral control represents an important factor in the driving process and for traffic safety. Its impairment by reduced awareness must be taken into account for a complete picture. Concerning the neglect of other error sources than the perception level in the error model, we do not think that this is a major limiting factor for the accuracy at the moment. Although errors appear also at other levels such as processing or actuation, the perceptive error propagates through all levels of the driving task 
and we have no indications that the resolution of different levels would add to the predictive capabilities of the model.

\section{Acknowledgements}

The authors gratefully acknowledge funding by the TransAID project under the Horizon 2020 Framework Programme, Grant Agreement no. 723390. 


\section{Appendix - Model Parameter}

The parametrization scheme utilized for the simulations presented in Section 3 is a balanced scheme, where intermediate parameter values are chosen with respect to the originally considered parameter range. Table 1 shows the parametrizations for the different vehicle categories. Where the entry is not a single number, it has the format normal $(<\operatorname{mean}>,<\operatorname{std}>) ;[<\min >,<\max >]$, specifying a cut off Gaussian distribution for the vehicle instances in the corresponding category.

Table 3: Parametrization of vehicle models

\begin{tabular}{|c|c|c|c|c|}
\hline \multirow[t]{2}{*}{ Parameter Name } & \multirow{2}{*}{$\begin{array}{l}\text { Parameter } \\
\text { description }\end{array}$} & \multicolumn{3}{|c|}{ Parameter values } \\
\hline & & $\begin{array}{c}\text { MV } \\
\text { (CF Model } \\
\text { Krauss) } \\
\end{array}$ & $\begin{array}{c}\text { CV } \\
\text { (CF Model } \\
\text { ACC) }\end{array}$ & $\begin{array}{c}\text { CAV } \\
\text { (CF Model } \\
\text { ACC) }\end{array}$ \\
\hline sigma & $\begin{array}{l}\text { Driver } \\
\text { imperfection }\end{array}$ & $\begin{array}{c}\operatorname{normal}(0.2, \\
0.5) ;[0.0,1.0]\end{array}$ & 0.0 & 0.0 \\
\hline $\operatorname{tau}[s]$ & $\begin{array}{l}\text { Desired time } \\
\text { headway }\end{array}$ & $\begin{array}{c}\text { normal }(0.6, \\
0.5) ;[0.5,1.6]\end{array}$ & $\begin{array}{c}\operatorname{normal}(1.6,0.2) \\
{[1.3,1.8]}\end{array}$ & $\begin{array}{c}\operatorname{normal}(1.6,0.2) \\
{[1.3,1.8]}\end{array}$ \\
\hline $\operatorname{decel}\left[\mathrm{m} / \mathrm{s}^{2}\right]$ & $\begin{array}{l}\text { Preferred maximal } \\
\text { deceleration }\end{array}$ & $\begin{array}{c}\operatorname{normal}(3.5, \\
1.0) ;[2.0,4.5]\end{array}$ & $\begin{array}{c}\operatorname{normal}(3.0,1.0) \\
{[2.0,4.0]}\end{array}$ & $\begin{array}{c}\operatorname{normal}(3.0,1.0) \\
{[2.0,4.0]}\end{array}$ \\
\hline $\operatorname{accel}\left[\mathbf{m} / \mathbf{s}^{2}\right]$ & $\begin{array}{l}\text { Maximal } \\
\text { acceleration }\end{array}$ & $\begin{array}{c}\operatorname{normal}(2.0, \\
1.0) ;[1.0,3.5]\end{array}$ & $\begin{array}{c}\operatorname{normal}(1.5,1.0) \\
{[0.75,2.0]}\end{array}$ & $\begin{array}{c}\operatorname{normal}(1.5,1.0) \\
{[0.75,2.0]}\end{array}$ \\
\hline $\begin{array}{l}\text { emergencyDecel } \\
{\left[\mathbf{m} / \mathbf{s}^{2}\right]}\end{array}$ & $\begin{array}{l}\text { Physically } \\
\text { maximal } \\
\text { deceleration }\end{array}$ & 9.0 & 9.0 & 9.0 \\
\hline lcAssertive & $\begin{array}{l}\text { Lane-change } \\
\text { aggressiveness } \\
\text { (willingness to } \\
\text { accept lower gaps) }\end{array}$ & 1.3 & 1.0 & $\begin{array}{c}\operatorname{normal}(0.7,0.1) \\
{[0.6,0.8]}\end{array}$ \\
\hline $\begin{array}{l}\text { actionStepLength } \\
\text { [s] }\end{array}$ & $\begin{array}{l}\text { Interval length at } \\
\text { which vehicle } \\
\text { maneuver logic is } \\
\text { executed } \\
\text { ( reaction time) }\end{array}$ & 0.1 & 0.1 & 0.1 \\
\hline speedFactor & $\begin{array}{l}\text { Proportionality } \\
\text { factor for the } \\
\text { desired speed }\end{array}$ & $\begin{array}{c}\operatorname{normal}(1.1, \\
0.2) ;[0.8,1.2]\end{array}$ & 1.0 & 1.0 \\
\hline responseTime [s] & $\begin{array}{l}\text { Time to takeover } \\
\text { after request }\end{array}$ & - & 1.0 & $\begin{array}{c}\text { normal }(7,2.5) \text {; } \\
{[2,60]}\end{array}$ \\
\hline timeTillMRM & $\begin{array}{l}\text { Available lead } \\
\text { time to takeover }\end{array}$ & - & 1.0 & 10.0 \\
\hline initialAwareness & $\begin{array}{l}\text { Driver awareness } \\
\text { after takeover }\end{array}$ & - & $\begin{array}{c}\operatorname{normal}(0.5,0.3) \\
{[0.1,1.0]}\end{array}$ & $\begin{array}{c}\operatorname{normal}(0.5,0.3) \\
{[0.1,1.0]}\end{array}$ \\
\hline recoveryRate $\left[\mathrm{s}^{-1}\right]$ & $\begin{array}{l}\text { Relaxation rate of } \\
\text { post-ToC } \\
\text { awareness }\end{array}$ & - & $\begin{array}{c}\operatorname{normal}(0.2,0.1) \\
{[0.01,0.5]}\end{array}$ & $\begin{array}{c}\operatorname{normal}(0.2,0.1) \\
{[0.01,0.5]}\end{array}$ \\
\hline mrmDecel $\left[\mathbf{m} / \mathbf{s}^{2}\right]$ & $\begin{array}{l}\text { Constant } \\
\text { deceleration rate } \\
\text { during MRM }\end{array}$ & - & 3.0 & 3.0 \\
\hline
\end{tabular}




\section{References}

Alkim, T. P., Bootsma, G., \& Hoogendoorn, S. P. (2007). Field Operational Test "The Assisted Driver." In 2007 IEEE Intelligent Vehicles Symposium (pp. 1198-1203). https://doi.org/10.1109/IVS.2007.4290281

Bansal, M., Krizhevsky, A., \& Ogale, A. (2018). ChauffeurNet: Learning to Drive by Imitating the Best and Synthesizing the Worst. ArXiv:1812.03079 [Cs]. Retrieved from http://arxiv.org/abs/1812.03079

Eriksson, A., \& Stanton, N. A. (2017). Driving Performance After Self-Regulated Control Transitions in Highly Automated Vehicles. Human Factors: The Journal of the Human Factors and Ergonomics Society, 001872081772877. https://doi.org/10.1177/0018720817728774

ERTRAC Working Group, "Connectivity and Automated Driving." (2017). Automated Driving Roadmap (No. Version 7.0). ERTRAC.

ETSI TS 103301 - Intelligent Transport Systems (ITS); Vehicular Communications; Basic Set of Applications; Facilities layer protocols and communication requirements for infrastructure services. (2016). (No. v1.1.1).

Evangelos Mintsis, Leonhard Luecken, Kallirroi Porfyri, Dimitris Koutras, Xiaoyun Zhang, Michele Rondinone, ... Evangelos Mitsakis. (2018). TransAID Deliverable 3.1 Modelling, simulation and assessment of vehicle automations and automated vehicles' driver behaviour in mixed traffic. Retrieved from https://www.transaid.eu/wpcontent/uploads/2017/Deliverables/WP3/TransAID_D3.1_Modelling-simulationand-assessment-of-vehicle-automations.pdf

Favarò, F., Eurich, S., \& Nader, N. (2018). Autonomous vehicles' disengagements: Trends, triggers, and regulatory limitations. Accident Analysis \& Prevention, 110, 136-148. https://doi.org/10.1016/j.aap.2017.11.001

Fuller, R. (2005). Towards a general theory of driver behaviour. Accident Analysis \& Prevention, 37(3), 461-472.

Gardiner, C. (2009). Stochastic methods (Vol. 4). springer Berlin.

Gettman, D., \& Head, L. (2003). Surrogate safety measures from traffic simulation models. Transportation Research Record: Journal of the Transportation Research Board, (1840), 104-115.

Gipps, P. G. (1981). A behavioural car-following model for computer simulation. Transportation Research Part B: Methodological, 15(2), 105-111. http://dx.doi.org/10.1016/0191-2615(81)90037-0

Gold, C., Damböck, D., Lorenz, L., \& Bengler, K. (2013). “Take over!” How long does it take to get the driver back into the loop? Proceedings of the Human Factors and Ergonomics Society Annual Meeting, 57(1), 1938-1942. https://doi.org/10.1177/1541931213571433

Gold, C., Körber, M., Lechner, D., \& Bengler, K. (2016). Taking over control from highly automated vehicles in complex traffic situations: the role of traffic density. Human Factors, 58(4), 642-652.

Kesting, A., \& Treiber, M. (2013). Traffic flow dynamics: data, models and simulation. No. Book, Whole)(Springer Berlin Heidelberg, Berlin, Heidelberg, 2013). 
Klunder, G., Li, M., \& Minderhoud, M. (2009). Traffic Flow Impacts of Adaptive Cruise Control Deactivation and (Re)Activation with Cooperative Driver Behavior. Transportation Research Record: Journal of the Transportation Research Board, 2129, 145-151. https://doi.org/10.3141/2129-17

Krauß, S. (1998). Microscopic modeling of traffic flow: Investigation of collision free vehicle dynamics (PhD Thesis). Universitat zu Koln.

Krauß, S., Wagner, P., \& Gawron, C. (1997). Metastable states in a microscopic model of traffic flow. Physical Review E, 55(5), 5597.

Liu, H., Kan, X. (David), Shladover, S. E., Lu, X.-Y., \& Ferlis, R. E. (2018). Impact of cooperative adaptive cruise control on multilane freeway merge capacity. Journal of Intelligent Transportation $\quad$ Systems, 22(3), 263-275. https://doi.org/10.1080/15472450.2018.1438275

Lopez, P. A., Behrisch, M., Bieker-Walz, L., Erdmann, J., Flötteröd, Y.-P., Hilbrich, R., ... Wießner, E. (2018). Microscopic Traffic Simulation using SUMO. In 2018 21st International Conference on Intelligent Transportation Systems (ITSC) (pp. 25752582). IEEE.

Lu, Z., Coster, X., \& de Winter, J. (2017). How much time do drivers need to obtain situation awareness? A laboratory-based study of automated driving. Applied Ergonomics, 60, 293-304. https://doi.org/10.1016/j.apergo.2016.12.003

Lücken, L. (2019). Resolving collisions for the Gipps Car-Following Model. ArXiv:1902.04927 [Stat]. Retrieved from https://arxiv.org/abs/1902.04927

Maerivoet, S., Akkermans, L., Carlier, K., Flötteröd, Y.-P., Lücken, L., Alms, R., ... Schindler, J. (2019). TransAID Deliverable 4.2 - Preliminary Simulation and Assessment of enhanced traffic management measures. Retrieved from https://www.transaid.eu/wpcontent/uploads/2017/Deliverables/WP4/TransAID_D4.2_Preliminary-simulationand-assessment-of-enhanced-traffic-management-measures.pdf

Merat, N., Jamson, A. H., Lai, F. C. H., Daly, M., \& Carsten, O. M. J. (2014). Transition to manual: Driver behaviour when resuming control from a highly automated vehicle. Transportation Research Part F: Traffic Psychology and Behaviour, 27, 274-282. https://doi.org/10.1016/j.trf.2014.09.005

Milanés, V., \& Shladover, S. E. (2014). Modeling cooperative and autonomous adaptive cruise control dynamic responses using experimental data. Transportation Research Part C: Emerging Technologies, 48, 285-300. https://doi.org/10.1016/j.trc.2014.09.001

Milanés, V., Shladover, S. E., Spring, J., Nowakowski, C., Kawazoe, H., \& Nakamura, M. (2014). Cooperative Adaptive Cruise Control in Real Traffic Situations. IEEE Transactions on Intelligent Transportation Systems, 15(1), 296-305. https://doi.org/10.1109/TITS.2013.2278494

Pauwelussen, J., \& Feenstra, P. J. (2010). Driver Behavior Analysis During ACC Activation and Deactivation in a Real Traffic Environment. IEEE Transactions on Intelligent Transportation Systems, 11(2), 329-338. https://doi.org/10.1109/TITS.2010.2043099 
Pauwelussen, J., \& Minderhoud, M. (2008). The effects of deactivation and (re)activation of ACC on driver behaviour analyzed in real traffic. In 2008 IEEE Intelligent Vehicles Symposium (pp. 257-262). https://doi.org/10.1109/IVS.2008.4621309

PTOLEMUS Consulting Group. (2017). Autonomous Vehicle Global Study - Free Abstract. Retrieved from www.ptolemus.com

Saifuzzaman, M., Zheng, Z., Haque, M. M., \& Washington, S. (2015). Revisiting the TaskCapability Interface model for incorporating human factors into car-following models. Transportation Research Part B: Methodological, 82, 1-19.

Todosiev, E. P. (1963). The action point model of the driver-vehicle system ( $\mathrm{PhD}$ Thesis). The Ohio State University.

Viti, F., Hoogendoorn, S. P., Alkim, T. P., \& Bootsma, G. (2008). Driving behavior interaction with ACC: results from a Field Operational Test in the Netherlands. In 2008 IEEE Intelligent Vehicles Symposium (pp. 745-750). https://doi.org/10.1109/IVS.2008.4621199

Wijbenga, A., Mintsis, E., Vreeswijk, J., Correa, A., Luecken, L., Schindler, J., ... Markowski, R. (2018). Scenario definitions and modelling requirements (No. TransAID Deliverable D2.2).

Wilson, R. E. (2001). An analysis of Gipps's car-following model of highway traffic. IMA Journal of Applied $\quad$ Mathematics, 509. https://doi.org/10.1093/imamat/66.5.509

Xiao, L., Wang, M., Schakel, W., \& van Arem, B. (2018). Unravelling effects of cooperative adaptive cruise control deactivation on traffic flow characteristics at merging bottlenecks. Transportation Research Part C: Emerging Technologies, 96, 380-397. https://doi.org/10.1016/j.trc.2018.10.008

Xiao, L., Wang, M., \& van Arem, B. (2017). Realistic Car-Following Models for Microscopic Simulation of Adaptive and Cooperative Adaptive Cruise Control Vehicles. Transportation Research Record: Journal of the Transportation Research Board, 2623, 1-9. https://doi.org/10.3141/2623-01

Xin, W., Hourdos, J., Michalopoulos, P., \& Davis, G. (2008). The less-than-perfect driver: a model of collision-inclusive car-following behavior. Transportation Research Record: Journal of the Transportation Research Board, (2088), 126-137.

Young, M. S., \& Stanton, N. A. (2002). Malleable Attentional Resources Theory: A New Explanation for the Effects of Mental Underload on Performance. Human Factors, 44(3), 365-375. https://doi.org/10.1518/0018720024497709 